\title{
EULER CHARACTERISTIC OF FREDHOLM QUASICOMPLEXES
}

\author{
N. TARKHANOV
}

\begin{abstract}
By quasicomplexes are usually meant perturbations of complexes small in some sense. Of interest are not only perturbations within the category of complexes but also those going beyond this category. A sequence perturbed in this way is no longer a complex, and so it bears no cohomology. We show how to introduce Euler characteristic for small perturbations of Fredholm complexes. The paper is to appear in Funct. Anal. and its Appl., 2006.
\end{abstract}

\section{Contents}

1. Introduction

2. Fredholm quasicomplexes

3. Reduction to a complex 4

4. Euler characteristic 6

References

\section{Introduction}

In the category of Hilbert spaces, a complex $(L, d)$ is given by a sequence of Hilbert spaces $L^{i}, i \in \mathbb{Z}$, and continuous linear mappings $d^{i}: L^{i} \rightarrow L^{i+1}$, such that $d^{i} \circ d^{i-1}=0$.

We will write it simply $L$ when no confusion can arise. When considering bounded complexes we can certainly assume that $L^{i}=0$ for $i$ different from $0,1, \ldots, N$, for if not, we shift the indexing.

Let $Z^{i}(L)$ and $B^{i}(L)$ stand for the spaces of cocycles and coboundaries of a complex $L$ at step $i$. Obviously, $B^{i}(L)$ is a vector subspace of $Z^{i}(L)$. A complex $L$ is said to be Fredholm if its cohomology $H^{i}(L)=Z^{i}(L) / B^{i}(L)$ is finite dimensional at each step $i$. For any Fredholm complex $L$, the Euler characteristic $\chi(L)$ is defined by

$$
\chi(L)=\sum_{i=0}^{N}(-1)^{i} \operatorname{dim} H^{i}(L) .
$$

Assume that $d+V$ is a perturbation of the differential $d$, with $V$ "small" enough. By "small" are meant appropriate ideals of compact operators. Then the square $(d+V)^{2}=d V+V d+V^{2}$ need not be zero but merely "small" along with $V$. The sequence $(L, d+V)$ is no longer a complex, and so it bears no cohomology. While a

Date: May 26, 2006.

2000 Mathematics Subject Classification. Primary 55U05; Secondary 58J10, 19 K56.

Key words and phrases. Essential complexes, Fredholm complexes, Euler characteristic. 
topological index might still be defined for $(L, d+V)$, the Euler characteristic can not.

As but one example of this we show the sequence of covariant differentiations related to a connection of a vector bundle $F$ over a $C^{\infty}$ compact manifold. In this case the compositions $d^{i} \circ d^{i-1}$ are identified with the curvature of the connection, which is a smooth differential form of degree 2 with values in the endomorphisms of $F$.

In contrast to the Euler characteristic, the signature invariants of Fredholm quasicomplexes are easier to define, cf. [Mis00].

\section{Fredholm QUASICOMPLEXES}

From the point of view of analysis, quasicomplexes seem to be much more natural objects than complexes. Indeed, "small" perturbations of Fredholm operators do not affect the Fredholm property. In particular, perturbing a single Fredholm operator by compact operators leads to a Fredholm operator. It would be desirable to have the same property for Fredholm complexes but most of the perturbations lead out the class of 'complexes'. For example, perturbing an elliptic complex by lower order terms does not change the sequence of principal symbols which remains to be exact away from the zero section of the cotangent bundle. However, the operators no longer satisfy $d^{i} \circ d^{i-1}=0$, and so the standard theory does not apply to the deformed complex. We are thus lead to a class of sequences $L=\left(L^{i}, d^{i}\right)_{i \in \mathbb{Z}}$ bearing the property that the compositions $d^{i} \circ d^{i-1}$ are small in some reasonable sense.

Definition 2.1. By a (cochain) quasicomplex $(L, d)$ is meant any sequence of Fréchet spaces $L^{i}, i \in \mathbb{Z}$, and operators $d^{i} \in \mathcal{L}\left(L^{i}, L^{i+1}\right)$ satisfying $d^{i} \circ d^{i-1}=0$ modulo compact operators.

Denote by $\mathcal{K}(L, \tilde{L})$ the subspace of $\mathcal{L}(L, \tilde{L})$ consisting of compact operators. For $m_{1}, m_{2} \in \mathcal{L}(L, \tilde{L})$, we write $m_{1} \sim m_{2}$ if $m_{1}-m_{2} \in \mathcal{K}(L, \tilde{L})$.

Suppose $(L, d)$ and $(\tilde{L}, \tilde{d})$ are two quasicomplexes. By a cochain mapping of $(L, d)$ into $(\tilde{L}, \tilde{d})$ is meant any collection of operators $m^{i} \in \mathcal{L}\left(L^{i}, \tilde{L}^{i}\right), i \in \mathbb{Z}$, such that $\tilde{d}^{i} m^{i} \sim m^{i+1} d^{i}$ for all $i \in \mathbb{Z}$. In particular, the families $0=\left(0_{L^{i}}\right)_{i \in \mathbb{Z}}$ and $1=\left(1_{L^{i}}\right)_{i \in \mathbb{Z}}$ are cochain mappings of $(L, d)$ into itself, and so are all their compact perturbations.

Cochain mappings $\left(m_{0}^{i}\right)_{i \in \mathbb{Z}}$ and $\left(m_{1}^{i}\right)_{i \in \mathbb{Z}}$ of $(L, d)$ into $(\tilde{L}, \tilde{d})$ are said to be homotopic if there is a collection $h^{i} \in \mathcal{L}\left(L^{i}, \tilde{L}^{i-1}\right), i \in \mathbb{Z}$, with the property that $m_{1}^{i}-m_{0}^{i} \sim \tilde{d}^{i-1} h^{i}+h^{i+1} d^{i}$ for all $i \in \mathbb{Z}$.

The task is now to introduce the concept of a Fredholm quasicomplex. Recall that an operator $d \in \mathcal{L}(L, \tilde{L})$ in Fréchet spaces is Fredholm if and only if its image in the Calkin algebra $\mathcal{L}(L, \tilde{L}) / \mathcal{K}(L, \tilde{L})$ is invertible. Thus, the idea is to pass in a given quasicomplex to quotients modulo spaces of compact operators and require exactness. To this end, we make use of a functor $\phi_{\Sigma}$ studied by Putinar [Put82]. For complexes of pseudodifferential operators it specifies to what is known as complex of symbols.

For Fréchet spaces $L$ and $\Sigma$, set $\phi_{\Sigma}(L)=\mathcal{L}(\Sigma, L) / \mathcal{K}(\Sigma, L)$. Moreover, given any $d \in \mathcal{L}(L, \tilde{L})$, we define $\phi_{\Sigma}(d) \in \mathcal{L}\left(\phi_{\Sigma}(L), \phi_{\Sigma}(\tilde{L})\right)$ by the formula

$$
\phi_{\Sigma}(d)(m+\mathcal{K}(\Sigma, L))=d m+\mathcal{K}(\Sigma, \tilde{L})
$$


for $m \in \mathcal{L}(\Sigma, L)$. Clearly, this operator is well defined. It is easily seen that $\phi_{\Sigma}\left(d^{2} d^{1}\right)=\phi_{\Sigma}\left(d^{2}\right) \phi_{\Sigma}\left(d^{1}\right)$ for all $d^{1} \in \mathcal{L}\left(L^{1}, L^{2}\right)$ and $d^{2} \in \mathcal{L}\left(L^{2}, L^{3}\right)$. If $1_{L}$ is the identity operator on $L$, then $\phi_{\Sigma}\left(1_{L}\right)$ is the identity operator on $\phi_{\Sigma}(L)$. These remarks show that $\phi_{\Sigma}$ is actually a covariant functor in the category of Fréchet spaces.

The crucial fact is that $\phi_{\Sigma}$ vanishes on compact operators, for every Fréchet space $\Sigma$. Conversely, if $d \in \mathcal{L}(L, \tilde{L})$ and $\phi_{\Sigma}(d)=0$ for any Fréchet space $\Sigma$, then $d \in \mathcal{K}(L, \tilde{L})$. Indeed, taking $\Sigma=L$, we deduce from

$$
\begin{aligned}
\phi_{L}(d)\left(1_{L}+\mathcal{K}(L, L)\right) & =d+\mathcal{K}(L, \tilde{L}) \\
& =\mathcal{K}(L, \tilde{L})
\end{aligned}
$$

that $d \in \mathcal{K}(L, \tilde{L})$.

Note that if $(L, d)$ is an arbitrary quasicomplex, then $\left(\phi_{\Sigma}(L), \phi_{\Sigma}(d)\right)$ is a complex, for each Fréchet space $\Sigma$. Thus, the functor $\phi_{\Sigma}$ transforms quasicomplexes into ordinary complexes. Furthermore, cochain mappings of quasicomplexes transform under $\phi_{\Sigma}$ into cochain mappings of complexes, and $\phi_{\Sigma}$ preserves the homotopy classes of cochain mappings.

Definition 2.2. A quasicomplex $(L, d)$ is called Fredholm if the associated complex $\left(\phi_{\Sigma}(L), \phi_{\Sigma}(d)\right)$ is exact, for each Fréchet space $\Sigma$.

Let $(L, d)$ and $(L, \tilde{d})$ be two quasicomplexes, such that $d^{i} \sim \tilde{d}^{i}$ for all $i \in \mathbb{Z}$. Then the complexes $\left(\phi_{\Sigma}(L), \phi_{\Sigma}(d)\right)$ and $\left(\phi_{\Sigma}(L), \phi_{\Sigma}(\tilde{d})\right)$ obviously coincide, for every Fréchet space $\Sigma$. Therefore, $(L, d)$ and $(L, \tilde{d})$ are simultaneously Fredholm. In other words, any compact perturbation of a Fredholm quasicomplex is a Fredholm quasicomplex.

Theorem 2.3. A bounded above quasicomplex $(L, d)$ is Fredholm if and only if the identity mapping of $(L, d)$ is homotopic to the zero one.

This theorem goes back at least as far as [Put82] wherein the designation 'essential complexes' is used for what we call 'quasicomplexes' here.

Proof. Necessity. Let $(L, d)$ be Fredholm and bounded above, i.e., $L^{i}=0$ for all but $i \leq N$. Our goal is to show that there are operators $\pi^{i} \in \mathcal{L}\left(L^{i}, L^{i-1}\right), i \in \mathbb{Z}$, such that

$$
d^{i-1} \pi^{i}+\pi^{i+1} d^{i}=1_{L^{i}}-c^{i}
$$

for all $i \in \mathbb{Z}$, where $c^{i} \in \mathcal{K}\left(L^{i}\right)$.

Set $\pi^{i}=0$ for all integers $i>N$. If $i=N$, then from the exactness of the complex $\left(\phi_{\Sigma}(L), \phi_{\Sigma}(d)\right), \Sigma=L^{N}$, at step $N$ if follows that there is an operator $\pi^{N} \in \mathcal{L}\left(L^{N}, L^{N-1}\right)$ such that $d^{N-1} \pi^{N} \sim 1_{L^{N}}$. Denoting by $c^{N}$ the difference $1_{L^{N}}-d^{N-1} \pi^{N}$, we thus get $c^{N} \in \mathcal{K}\left(L^{N}\right)$.

We now proceed by induction. Suppose we have already found mappings

$$
\begin{array}{lll}
\pi^{i}, & \pi^{i+1}, & \ldots ; \\
c^{i}, & c^{i+1}, & \ldots,
\end{array}
$$


such that the equality $(2.1)$ is satisfied at steps $i, i+1, \ldots$, for some $i \leq N$. Note that

$$
\begin{aligned}
d^{i-1}\left(1_{L^{i-1}}-\pi^{i} d^{i-1}\right) & =d^{i-1}-\left(1_{L^{i}}-c^{i}-\pi^{i+1} d^{i}\right) d^{i-1} \\
& =c^{i} d^{i-1}+\pi^{i+1} d^{i} d^{i-1} \\
& \sim 0
\end{aligned}
$$

by (2.1). From the exactness of $\left(\phi_{\Sigma}(L), \phi_{\Sigma}(d)\right)$, with $\Sigma=L^{i-1}$, at step $i-1$ it follows that there is $\pi^{i-1} \in \mathcal{L}\left(L^{i-1}, L^{i-2}\right)$ such that $d^{i-2} \pi^{i-1} \sim 1_{L^{i-1}}-\pi^{i} d^{i-1}$. Setting $c^{i-1}=1_{L^{i-1}}-\pi^{i} d^{i-1}-d^{i-2} \pi^{i-1}$, we obtain $c^{i-1} \in \mathcal{K}\left(L^{i-1}\right)$ and $(2.1)$ fulfilled at step $i-1$. This establishes the existence of solutions $\pi^{i}, c^{i}$ to (2.1) for each $i \in \mathbb{Z}$, i.e., the homotopy between the identity and zero cochain mappings of $(L, d)$.

Sufficiency. If the identity mapping $1=\left(1_{L^{i}}\right)_{i \in \mathbb{Z}}$ is homotopic to the zero mapping $0=\left(0_{L^{i}}\right)_{i \in \mathbb{Z}}$ on $(L, d)$, then the identity mapping on the cohomology $H^{i}\left(\phi_{\Sigma}(L), \phi_{\Sigma}(d)\right)$ vanishes for all $i \in \mathbb{Z}$. Hence, the complex $\left(\phi_{\Sigma}(L), \phi_{\Sigma}(d)\right)$ is exact for each Fréchet space $\Sigma$, as required.

Any solution $\pi^{i} \in \mathcal{L}\left(L^{i}, L^{i-1}\right), i \in \mathbb{Z}$, to $(2.1)$ is called a parametrix of quasicomplex $(L, d)$. Thus, Theorem 2.3 just amounts to saying that a bounded above quasicomplex is Fredholm if and only if it possesses a parametrix. Given any Fredholm quasicomplex $(L, d)$, if $f \in L^{i}$ satisfies $d^{i} f=0$, then $f=c^{i} f+d^{i-1} \pi^{i} f$, where $(L, \pi)$ is a parametrix for $(L, d)$ as in (2.1). In other words the operator $d^{i-1}$ has a right inverse $\pi^{i}$ on $Z^{i}(L)$ modulo compact operators. However, since the compositions $d^{i} d^{i-1}$ need not vanish for a quasicomplex $L$, the range of $d^{i-1}$ no longer lies in $Z^{i}(L)$. It follows that the usual cohomology does not make sense for $L$. The question on a proper substitute of the cohomology for quasicomplexes seems to be very subtle, cf. [ST99].

\section{Reduction to a COMplex}

Let $(L, d)$ be a Fredholm quasicomplex consisting of Hilbert spaces $L^{i}$ which are zero for all $i$ but $i=0,1, \ldots, N$, and operators $d^{i} \in \mathcal{L}\left(L^{i}, L^{i+1}\right)$ with $d^{i+1} \circ d^{i}$ compact.

These spaces and operators are fit together to form a sequence of Hilbert spaces of compact curvature, namely,

$$
0 \rightarrow L^{0} \stackrel{d^{0}}{\rightarrow} L^{1} \stackrel{d^{1}}{\rightarrow} \quad \ldots \quad \stackrel{d^{N-1}}{\rightarrow} L^{N} \rightarrow 0
$$

Theorem 3.1. For every Fredholm quasicomplex (3.1) there exist bounded operators $D^{i} \in \mathcal{L}\left(L^{i}, L^{i+1}\right)$ satisfying $D^{i}=d^{i}$ modulo compact operators of $\mathcal{K}\left(L^{i}, L^{i+1}\right)$ and $D^{i+1} D^{i}=0$ for all $i$.

Proof. Set $D^{N-1}=d^{N-1}$. The Laplacian

$$
\Delta^{N}=D^{N-1} D^{N-1 *}
$$

is a selfadjoint operator on $H^{N}$, and its kernel just amounts to the kernel of $D^{N-1 *}$. By Theorem 2.3, the latter operator $D^{N-1 *}$ has a left parametrix. In fact, the equality

$$
\pi^{N *} D^{N-1 *}=1_{L^{N}}-c^{N *}
$$

holds on $L^{N}$. Hence, the identity operator on $\operatorname{ker} D^{N-1 *}$ is compact. It follows that the kernel of $D^{N-1 *}$ is finite dimensional, and so $\Delta^{N}$ is Fredholm. 
By the abstract Hodge theory, there is a selfadjoint operator $G^{N} \in \mathcal{L}\left(L^{N}\right)$ mapping into the orthogonal complement of ker $\Delta^{N}$, such that $1_{L^{N}}=H^{N}+\Delta^{N} G^{N}$ on $L^{N}$, where $H^{N}$ is the orthogonal projection onto the finite-dimensional space $\operatorname{ker} \Delta^{N}=\operatorname{ker} D^{N-1 *}$.

The space $\operatorname{ker} D^{N-1 *}$ is thus an obstruction to the existence of a right fundamental solution for $D^{N-1}$. The operator $\Phi^{N}=D^{N-1 *} G^{N}$ is a special right parametrix for $D^{N-1}$ in $\mathcal{L}\left(L^{N}, L^{N-1}\right)$.

We now show that $P^{N-1}=1_{L^{N-1}}-\Phi^{N} D^{N-1}$ is an orthogonal projection onto the kernel of $D^{N-1}$. To this end, we note that $P^{N-1}$ is the identity operator on the kernel of $D^{N-1}$, and

$$
\begin{aligned}
D^{N-1} P^{N-1} & =D^{N-1}-\Delta^{N} G^{N} D^{N-1} \\
& =D^{N-1}-\left(1_{L^{N}}-H^{N}\right) D^{N-1} \\
& =0
\end{aligned}
$$

for $H^{N} D^{N-1}=\left(D^{N-1 *} H^{N}\right)^{*}=0$. From this the desired conclusion follows.

In order to construct $D^{N-2}$ we consider the last fragment of sequence (3.1), namely

Set

$$
L^{N-2} \quad \stackrel{d^{N-2}}{\rightarrow} \quad L^{N-1} \quad \stackrel{D^{N-1}}{\rightarrow} L^{N}
$$

then $D^{N-2} \in \mathcal{L}\left(L^{N-2}, L^{N-1}\right)$ satisfies

$$
D^{N-2}=P^{N-1} d^{N-2}
$$

$$
\begin{aligned}
D^{N-1} D^{N-2} & =D^{N-1} P^{N-1} d^{N-2} \\
& =0
\end{aligned}
$$

and

$$
\begin{aligned}
D^{N-2} & =\left(1_{L^{N-1}}-\Phi^{N} d^{N-1}\right) d^{N-2} \\
& =d^{N-2}
\end{aligned}
$$

modulo compact operators in $\mathcal{K}\left(L^{N-2}, L^{N-1}\right)$, as desired.

We now restrict ourselves to the suitably modified preceding fragment of the sequence (3.1), i.e.,

$$
L^{N-3} \stackrel{d^{N-3}}{\rightarrow} L^{N-2} \quad \stackrel{D^{N-2}}{\rightarrow} L^{N-1} .
$$

The Laplacian $\Delta^{N-1}=D^{N-1 *} D^{N-1}+D^{N-2} D^{N-2 *}$ is a selfadjoint operator in $\mathcal{L}\left(L^{N-1}\right)$, whose kernel is obviously $\operatorname{ker} D^{N-1} \cap \operatorname{ker} D^{N-2 *}$. Our next goal is to prove that this kernel is of finite dimension. To this end, we observe that the equality

$$
D^{N-2} \pi^{N-1}+\pi^{N} D^{N-1}=1_{L^{N-1}}-C^{N-1}
$$

holds for some compact operator $C^{N-1} \in \mathcal{K}\left(L^{N-1}\right)$, since both $D^{N-1}-d^{N-1}$ and $D^{N-2}-d^{N-2}$ are compact. Hence, the identity operator on the cohomology $H^{N-1}(L, D)$ is compact, and so the dimension of $H^{N-1}(L, D)$ is finite. Since the natural embedding ker $\Delta^{N-1} \hookrightarrow H^{N-1}(L, D)$ is injective, we immediately deduce that the kernel of $\Delta^{N-1}$ is finite dimensional, too. This shows that the Laplacian $\Delta^{N-1}$ is Fredholm.

By the abstract Hodge theory, there is a selfadjoint operator $G^{N-1} \in \mathcal{L}\left(L^{N-1}\right)$ which maps into the orthogonal complement of ker $\Delta^{N-1}$ and fulfills

$$
1_{L^{N-1}}=H^{N-1}+\Delta^{N-1} G^{N-1}
$$


on $L^{N-1}$, where $H^{N-1}$ is the orthogonal projection onto the finite-dimensional space ker $\Delta^{N-1}$.

We claim that $D^{N-1} G^{N-1}=G^{N} D^{N-1}$. To prove this, pick an arbitrary element $u \in L^{N-1}$. Then

$$
D^{N-1} u=D^{N-1} D^{N-1 *} D^{N-1} G^{N-1} u
$$

on the one hand, and

$$
D^{N-1} u=D^{N-1} D^{N-1 *} G^{N} D^{N-1} u
$$

on the other hand. Hence it follows that $\Delta^{N}\left(D^{N-1} G^{N-1} u-G^{N} D^{N-1} u\right)=0$, and since $D^{N-1} G^{N-1} u-G^{N} D^{N-1} u$ is orthogonal to ker $\Delta^{N}$ we conclude that $D^{N-1} G^{N-1} u-G^{N} D^{N-1} u=0$, as desired.

The composition $\Phi^{N-1}=D^{N-2 *} G^{N-1}$ is thus an operator in $\mathcal{L}\left(L^{N-1}, L^{N-2}\right)$ satisfying the homotopy equation

$$
\Phi^{N} D^{N-1}+D^{N-2} \Phi^{N-1}=1_{L^{N-1}}-H^{N-1} .
$$

In other words, the pair $\left\{\Phi^{N-1}, \Phi^{N}\right\}$ is a special parametrix at steps $N-1$ and $N$ for the sequence (3.1).

To construct $D^{N-3}$ we can now argue in the same way as in the construction of $D^{N-2}$. Namely, let us show that $P^{N-2}=1_{L^{N-2}}-\Phi^{N-1} D^{N-2}$ is an orthogonal projection onto the kernel of $D^{N-2}$. To this end, we note that $P^{N-2}$ is the identity operator on the kernel of $D^{N-2}$, and

$$
\begin{aligned}
D^{N-2} P^{N-2} & =D^{N-2}-D^{N-2} \Phi^{N-1} D^{N-2} \\
& =D^{N-2}-\left(1_{L^{N-1}}-H^{N-1}-\Phi^{N} D^{N-1}\right) D^{N-2} \\
& =0,
\end{aligned}
$$

for $H^{N-1} D^{N-2}=\left(D^{N-2 *} H^{N-1}\right)^{*}=0$. From this the desired conclusion readily follows.

Set

$$
D^{N-3}=P^{N-2} d^{N-3}
$$

then $D^{N-3} \in \mathcal{L}\left(L^{N-3}, L^{N-2}\right)$ satisfies

$$
\begin{aligned}
D^{N-2} D^{N-3} & =D^{N-2} P^{N-2} d^{N-3} \\
& =0
\end{aligned}
$$

and

$$
\begin{aligned}
D^{N-3} & =\left(1_{L^{N-2}}-\Phi^{N-1} D^{N-2}\right) d^{N-3} \\
& =\left(1_{L^{N-2}}-\Phi^{N-1} d^{N-2}\right) d^{N-3} \\
& =d^{N-3}
\end{aligned}
$$

modulo compact operators in $\mathcal{K}\left(L^{N-3}, L^{N-2}\right)$, as desired.

We now proceed by induction, thus completing the proof, for the sequence (3.1) terminates.

\section{Euler characteristic}

Consider a Fredholm quasicomplex (3.1), with $L^{i}$ being Hilbert spaces. By Theorem 3.1, there are operators $D^{i} \in \mathcal{L}\left(L^{i}, L^{i+1}\right)$, such that $D^{i}=d^{i}$ modulo 
compact operators of $\mathcal{K}\left(L^{i}, L^{i+1}\right)$ and $D^{i+1} D^{i}=0$ for all $i$. We thus arrive at a Fredholm complex

$$
0 \rightarrow L^{0} \stackrel{D^{0}}{\rightarrow} L^{1} \stackrel{D^{1}}{\rightarrow} \ldots \stackrel{D^{N-1}}{\rightarrow} L^{N} \rightarrow 0
$$

the latter being a consequence of the fact that Fredholm quasicomplexes are stable under compact perturbations.

Definition 4.1. By the Euler characteristic of Fredholm quasicomplex (3.1) is meant $\chi(L, d):=\chi(L, D)$.

The question arises whether this definition is correct, i.e., independent of the particular choice of complex (4.1).

Theorem 4.2. Suppose that $\left(L, D_{1}\right)$ and $\left(L, D_{2}\right)$ be two complexes with the property that $D_{1}^{i}=d^{i}$ and $D_{2}^{i}=d^{i}$ modulo compact operators of $\mathcal{K}\left(L^{i}, L^{i+1}\right)$. Then $\chi\left(L, D_{1}\right)=\chi\left(L, D_{2}\right)$.

Proof. We split $L$ into the sum

$$
L=L^{\text {even }} \oplus L^{\text {odd }}
$$

with $L^{\text {even }}=\oplus L^{2 i}$ and $L^{\text {odd }}=\oplus L^{2 i+1}$. Let now

$$
\begin{aligned}
& \left(D_{1}+D_{1}^{*}\right)_{e}: L^{\text {even }} \rightarrow L^{\text {odd }}, \\
& \left(D_{2}+D_{2}^{*}\right)_{e}: L^{\text {even }} \rightarrow L^{\text {odd }}
\end{aligned}
$$

denote the restrictions of $D_{1}+D_{1}^{*}$ and $D_{2}+D_{2}^{*}$ to $L^{\text {even }}$, respectively. The abstract Hodge theory allows one to conclude that the index of $\left(D_{1}+D_{1}^{*}\right)_{e}$ is equal to $\chi\left(L, D_{1}\right)$ and the index of $\left(D_{2}+D_{2}^{*}\right)_{e}$ is equal to $\chi\left(L, D_{2}\right)$. Since the Fredholm operators $\left(D_{1}+D_{1}^{*}\right)_{e}$ and $\left(D_{2}+D_{2}^{*}\right)_{e}$ differ from each other by a compact operator, their indices actually coincide. It follows that $\chi\left(L, D_{1}\right)=\chi\left(L, D_{2}\right)$, which completes the proof.

Thus, the Fredholm quasicomplexes of operators in Hilbert spaces bear welldefined Euler characteristics.

Note that the Euler characteristic of a Fredholm quasicomplex can be evaluated by a crude formula

$$
\chi(L, d)=\sum_{i=0}^{N}(-1)^{i} \operatorname{tr}\left(1_{L^{i}}-d^{i-1} \pi^{i}-\pi^{i+1} d^{i}\right)
$$

where $\left\{\pi^{i}\right\}$ is a parametrix of $(L, d)$ modulo trace class operators. This follows by arguments in [Fed91, p. 203]. 


\section{REFERENCES}

[Fed91] Fedosov, B. V., Index Theorems, In: Modern Problems of Mathematics. Fundamental Directions. Vol. 65, VINITI, Moscow, 1991, 165-268.

[Mis00] Mishchenko, A. S., The theory of almost algebraic Poincaré complexes, In: Proc. Steklov Inst. Math. (2000), no. 4 (231), 281-307.

[Put82] Putinar, M., Some invariants for semi-Fredholm systems of essentially commuting operators, J. Operator Theory 8 (1982), 65-90.

[ST99] Schulze, B.-W., and TARKhanov, N., Elliptic complexes of pseudodifferential operators on manifolds with edges, In: Advances in Partial Differential Equations, WileyVCH, Berlin et al., 1999, 287-431.

[Tar95] Tarkhanov, N., Complexes of Differential Operators, Kluwer Academic Publishers, Dordrecht, NL, 1995.

(Nikolai Tarkhanov) Universität Potsdam, Institut für Mathematik, Postfach 6015 53, 14415 Potsdam, Germany

E-mail address: tarkhanov@math.uni-potsdam.de 